\title{
Effect of Nitridation Properties for High Quality GaN Deposition on Amorhpous Quartz Glass at Low Temperature Via ECR-PEMOCVD
}

\author{
Dong Zhang ${ }^{1}$, Zhenhe $\mathrm{Ju}^{1}$, Yucai $\mathrm{Li}^{1}$, Shiwei Song ${ }^{1}$, Jian Wang ${ }^{1}$, Gang Wang ${ }^{1}$, \\ Zhenya Guo ${ }^{2}$ \\ ${ }^{1}$ School of Renewable Energy, Shenyang Institute of Engineering, Shenyang, \\ China \\ 2 Jinzhou Power Supply Branch, State Grid Liaoning Electric Power Supply Co. \\ Ltd., Jinzhou, China \\ ambitious211@163.com
}

\begin{abstract}
GaN-based solar cells fabricating greatly depends on the using of cheap substrate and the high quality of the as-grown GaN films. The high c-axis preferred orientation GaN films were prepared on amorphous quartz glass substrate using self developed electron cyclotron resonance plasma enhanced metal-organic chemical vapor deposition (ECR-PEMOCVD) system. The properties of the GaN films were characterized by in situ Reflection High Energy Electron Diffraction (RHEED), X-ray diffraction (XRD), Transmission Spectra and photo-luminescence (PL) spectra. The results show that concentric diffraction RHEED rings demonstrated the successful nitriding of conventional amorhpous quartz glass substrates at low temperature of the $430^{\circ} \mathrm{C}$, and the temperature is far below the strain point of glass. And the nitridation with as-grown GaN films deposition exhibited stronger c-axis orientation, and the average grain size increased significantly. But which were increased the nitriding time to $30 \mathrm{~min}$, and the as-grown films show the deposition deteriorated under the condition, which means that the quality of GaN deposition depends on the nitriding time, and the experiment results show the 15 min was proved to be the optimum time.
\end{abstract}

Keywords: GaN films; glass substrate; nitridation; ECR-PEMOCVD system

\section{Introduction}

$\mathrm{GaN}$ film is as an important semiconductor material with the wide direct band gap, which can be made the high-efficiency blue or green light-emitting diodes (LED) and electron apparatus with high quality and frequency [1-5]. Currently, the exploitation of full-color display GaN based LED and white LED products are the research and development hot spot in global semiconductor, which also have become the most important application field. GaN based materials are mostly prepared on Sic, Si and sapphire single crystal substrates, and which have been greatly investigated for their application in LED, laser diodes (LD) and photo-detectors [6-13]. Due to wide band-gap and some excellent properties, such as good mobility, high saturated drift velocity, high mechanical and thermal stability and high transparency, and the GaN films for its alloys are also useful for fabricating high-efficiency soar cells. And some papers have designed $\mathrm{GaN} / \mathrm{InGaN}$ solar cells on sapphire substrates using MOCVD method. The cell reported has open-circuit voltages up to $2.4 \mathrm{~V}$ and internal quantum efficiency as high as $60 \%$. One particular difficulty in the application of $\mathrm{GaN}$ films deposition on solar cells is the unavailability of large cheap substrate. So the low cost quartz glass is preferred substrate 
material and how to achieve high quality GaN films on amorphous quartz glass is the key problem.

In recent years, with the large-capacity data transmission requirements, the as-grown GaN films are increasingly demanding for high-frequency SAW devices. And they are developing from the initial MHz-level to the present GHz-level. From a developmental point of view, the SAW filter whose working frequencies exceeds $1 \mathrm{GHz}$ has an extreme range of needs in the future of the high-frequency radio communications, while the SAW filter whose working frequencies exceeds $2.5 \mathrm{GHz}$ will reach the limits of lithography in the request for electrode width of right inter-digital transducer. In order to improve the operating frequency, SAW filters need to further increase the phase velocity, which can be realized by depositing $\mathrm{GaN}$ on the substrate of high phase velocity. Sound propagational speed of diamond is the fastest in all media and from the speed of sound to consider it is the best transaudient material, but it does not have piezoelectric properties. Therefore, the piezoelectric property films of $\mathrm{GaN}$ deposited on the surface of diamond, this GaN/diamond structure can couple surface acoustic wave with the surface of diamond. And the wave can transmit at the speed of approximate diamond audio transmission, thereby which are effectively improving the operating frequency of SAW devices. In addition, the highest thermal conductivity of diamond, and its good mechanical properties, chemical stability, frequency stability and excellent temperature stability, which make diamond as the ideal high-frequency SAW filter substrate material. It predicted that if further improve the preparation process, the diamond film SAW devices can be achieved frequency of $10 \mathrm{GHz}$ or even higher. Diamond film with high-frequency SAW filters are not only in frequency but in power durability and high-fidelity transmission superior to the traditional SAW filters. For low loss, high-frequency SAW filters, high-standard requirements are reflected in the electrical and mechanical coupling coefficient, frequency-temperature coefficient and transmission attenuation. These are subjected to the material properties of $\mathrm{GaN}$; therefore the high-quality GaN thin film is the basis of preparation of the device.

At present, $\mathrm{ZnO} /$ diamond structure has effectively improved the operating frequency of SAW filters. We also serve GaN/diamond structure as a technology choice, because of the advantages of $\mathrm{GaN}$ velocity $(5660 \mathrm{~m} / \mathrm{s}$, close to the diamond) higher than $\mathrm{ZnO}$ materials, piezoelectric properties, high thermal conductivity and high chemical stability. That is one of the hot piezoelectric materials to prepare high-power high-frequency SAW devices in recent years. Therefore, if using the $\mathrm{GaN} /$ diamond structure, it is superior to $\mathrm{ZnO} /$ diamond structure. There are several unique advantages in GaN/diamond multilayer structure. First of all, the GaN material SAW (surface acoustic wave) phase velocity is almost twice the $\mathrm{ZnO}$ materials. Therefore, GaN/ diamond multilayer structure surface acoustic wave phase speed is higher than the $\mathrm{ZnO}$ / diamond, and thus the width of the IDT with the same electrode can achieve higher frequencies; The second, the difference between GaN and diamond phase-velocity is small, and GaN/Diamond multi-layer structure is showing a small velocity dispersion, which is phase velocity changes little with a different frequency.

$\mathrm{ZnO} /$ diamond/Si structure has effectively improved the operating frequency of SAW filters, but this structure to use most of the applications of diamond is $10 \mathrm{~nm}$ to $100 \mathrm{~nm}$ of the diamond film, which is deposited by chemical vapor deposition (CVD) method. Due to the presence of the silicon substrate, this structure is difficult to play a diamond high thermal conductivity. From a practical point of view, how to improve the power persistence of SAW filters is still the key problem. GaN/self-sustaining diamond structure can make full use of the performance of high thermal conductivity of diamond with its high surface acoustic wave velocity. In order to obtain high quality surface acoustic wave filters, the GaN film deposition on the self-sustaining diamond thick film is a good choice. 
There have invented the regular MOCVD technique to prepare the blue GaN based LED on silicon wafer under the inexpensive style. And some company have also prepared blue GaN based LED used ECR-PEMOCVD on conductive glass substrate in Australia, which could not stand high temperature [14-17]. Many study proved that the PE can be used to prepare the GaN based LED at low temperature, then operating apparatus at low temperature and using cheap substrate can reduce the LED's production cost. But in recent years, preparing GaN-based LED on metallic films has been causing a great sensation [18-22].

Currently, in terms of preparing GaN based LED, the regular MOCVD apparatus all use NH3 and TMGa are as the source, which can't stand high temperature. And the high temperature will easily result volatilisation of $\mathrm{N}$ and $\mathrm{In}$, which are hardly growing high-quality InGaN film with high component of $\operatorname{In}$ (generally under $25 \%$ ) and restricting applications in red -light and other long wavelength light of GaN-based LED [20-22].

In this article, we were using the ECR-PEMOCVD method to deposition the high c-axis preferred orientation GaN films on amorhpous quartz glass at low temperature. The crystalline and electrical properties of as-deposited films were investigated by reflection high energy electron diffraction (RHEED) in situ, X-ray diffraction (XRD), atomic force microscope (AFM) and Hall measurements. And the transmission spectra and photoluminescence (PL) is to understand the crystalline and optical properties.

\section{Experimental Procedure}

The deposition of high quality GaN is performed in self-developed ECR-PEMOCVD system equipped with RHEED monitoring in situ. High-purity N2 is introduced into the discharge chamber to directly provide $\mathrm{N}$ active particles. If directly exposing to ECR plasma discharge, Carbon-Hydrogen Groups may decompound, which can cause carbon contamination. So Trimethyl-gallium (TMGa) has relatively lower pyrolysis temperature, which is transported into the reactor by a gas-puffed circle $5 \mathrm{~cm}$ above the rotating substrate holder using high-purity $\mathrm{H} 2$ as carrier gas.

ECR-PEMOCVD low-temperature epitaxial method has a unique advantage. ECR microwave discharge is applied to meet the ECR condition of the electric field and magnetic field on the microwave, and through the acquisition of electronic and atomic collisions with gas molecules larger energy in ECR high density and high excited plasma discharge. When the magnetic field intensity is constant, the frequency of the cyclotron motion of the charged particle is independent of its velocity, and the relation between the magnetic induction intensity and the cyclotron vibration frequency is satisfied:

$$
\omega_{\mathrm{ce}}=e B / m_{e}
$$

Among them, $\mathrm{e}$ is the electron charge, $m_{e}$ is the electron mass, and $B$ is the magnetic field strength.

The ECR-PEMOCVD device can provide independent reactive nitrogen plasma source of large area uniform and high concentration, the growth temperature constraints, and which can provide the plasma particles in the reaction activation energy of surface growth required for migration, which have solved the problem of how to grow the key scientific problems of high quality GaN thin films at low temperature. The disadvantage factors of GaN as-grown films can be avoided by the high temperature preparation technology of conventional MOCVD. And the plasma pretreatment of the substrate between GaN buffer layer growths is to release the stress with lattice mismatch. In order to obtain the good interface extension, the GaN film and the glass substrate are produced by large growth, thereby which are reducing the dislocation density of $\mathrm{GaN}$ films and the background electron concentration, which can improve the quality and growth the migration rate of 
film crystals and can solve the key scientific problems of how to grow high quality GaN films on glass substrates.

By using the high pure nitrogen plasma, the conventional MOCVD and PEMBE method avoid the decomposition of as nitrogen source in the growth of III nitride films under high temperature. And by nitrogen plasma annealing method without high-energy ion damage model, in order to solve the control nitrogen volatilization in the annealing process, which are annealing on GaN thin films in situ, which can achieve stable GaN the key scientific problems.

Firstly of all, the substrates are degreased ultrasonically with acetone, ethanol and deionized water for 10min respectively. Then they are cleaned with deionized water. Finally, they are blown dry with N2 and store in sample chamber full of N2, which are ready for using. The quartz substrates were annealed for $120 \mathrm{~min}$ under the temperature of $100{ }^{\circ} \mathrm{C}$. The $\mathrm{GaN}$ films were deposited for $200 \mathrm{~nm}$ under the following condition: temperature of $430^{\circ} \mathrm{C}$, microwave power of $650 \mathrm{~W}, \mathrm{~N} 2$ gas flow rate of $70 \mathrm{sccm}, \mathrm{TMGa}$ gas flow rate of $0.4 \mathrm{sccm}$ and $\mathrm{TMGa}$ source temperature of $-12.6^{\circ} \mathrm{C}$.

Table 1. The Parameters of the As-Grown Films Preparation

\begin{tabular}{ccccccc}
\hline \hline Parameters & $\mathrm{T} /{ }^{\circ} \mathrm{C}$ & $\mathrm{H}_{2} / \mathrm{sccm}$ & $\mathrm{N}_{2} / \mathrm{sccm}$ & $\mathrm{TMGa} / \mathrm{sccm}$ & $\mathrm{t} / \mathrm{min}$ & $\mathrm{P} / \mathrm{W}$ \\
\hline \hline Cleaning & room temperature & 80 & 20 & 0 & 10 & 650 \\
Buffer layer & room temperature & 0 & 80 & 0.6 & 30 & 650 \\
GaN film & $\mathrm{a} / \mathrm{b} / \mathrm{c} / \mathrm{d}$ & 0 & 100 & 1.0 & 180 & 650 \\
\hline \hline
\end{tabular}

\section{Results and Discussion}

\subsection{Structural Properties}

The experiments were using the ECR-PEMOCVD method to deposition the high c-axis preferred orientation GaN films on amorhpous quartz glass at low temperature. The crystalline and electrical properties of as-deposited films were investigated by the X-ray diffraction (XRD) method. And the Figure 1 shows the XRD spectrum of GaN film deposited on quartz glass substrate. The dominant strong peak at about $34.56^{\circ}$ is from the $\mathrm{GaN}(002)$ plane and the far more weak peak at $73.56^{\circ}$ corresponding to plane (004) plane, which is the second order diffraction of plane (002), the experiment results indicate that the GaN films have very strong preferred orientation with the c-axis normal to the substrate. The others small peaks shown in the figure at nearly $44^{\circ}$ and $65^{\circ}$ are from the sample table. The X-ray diffraction measurement were carried out by XRD-600. A very wide and low intensity peak can be seen from $2 \theta$ about 20 to about 80 degree, which is caused by the diffraction of amorphous glass substrate. The dominant (0002) peak corresponding to C-axis diffraction indicates that $\mathrm{GaN}$ deposition on glass substrates is with $\mathrm{C}$-axis preferential orientation wurtzite-type structure. 


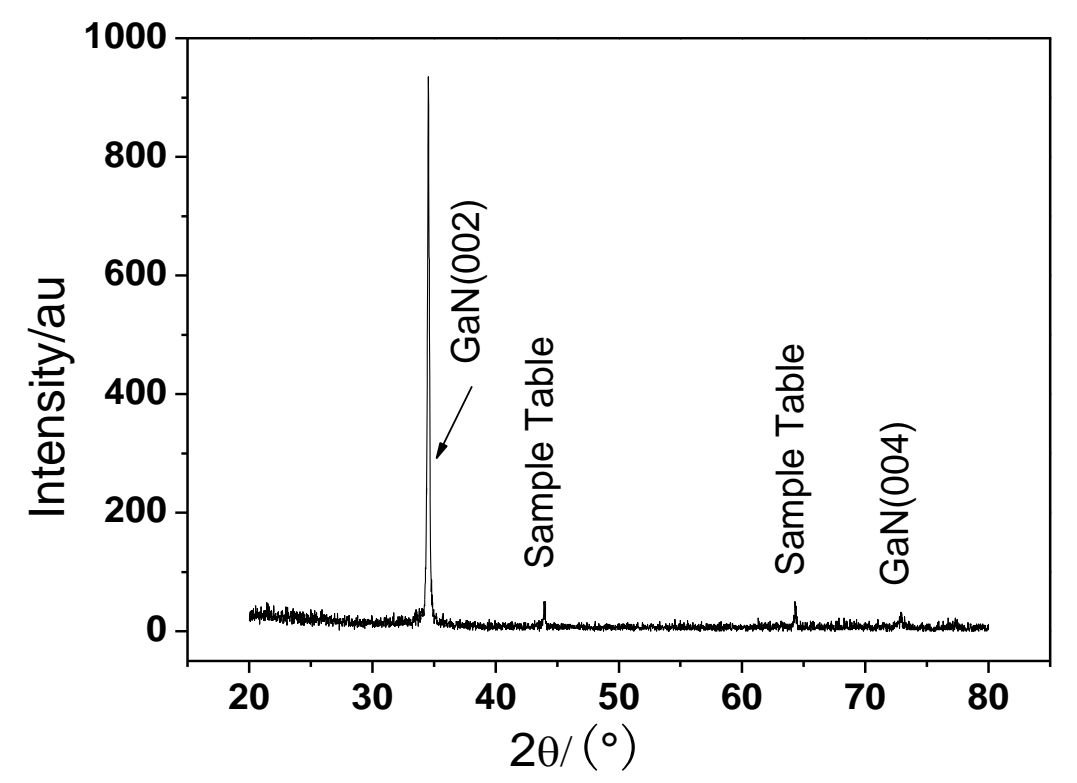

\section{Figure 1. XRD Spectrum of GaN Film Deposited On Quartz Glass Substrate}

\subsection{RHEED Measurement}

The experiments were using the ECR-PEMOCVD method to deposition the high c-axis preferred orientation GaN films on amorhpous quartz glass at low temperature. The crystalline properties of as-deposited films were investigated by reflection high energy electron diffraction (RHEED) in situ. And the Figure 2 is RHEED patterns of glass substrate before and after nitriding. As can be seen that the RHEED pattern of glass substrate, which is a bright spot without fixed pattern before nitridation. When the incident electron beam hit the surface of non-electrical conductive glass substrate, the electrons were collected up, which is caused from outside toward substrate electric field, which the later electrons were reflected and formed a bright spot. The rather weak concentric diffraction rings were recorded for glass substrate after nitriding for 5min, which are typical transmission diffraction patterns of polycrystalline films, this experiment results proved that the layer of nitridation was successfully formed. According to some researchers, the nitrided films were composed of an layer of silicon oxynitride ( $\mathrm{SiOxNy}$ ), which have reported a variation of $\mathrm{O}$ and $\mathrm{N}$ component ratio with different nitriding temperature, according to the surface region was transformed to Si3N4 completely at lower temperature about $25^{\circ} \mathrm{C}$ and silicon oxynitride was obtained at higher temperature. The RHEED pattern of glass substrate with longer nitriding time were also recorded, which did not showed any significant changed. The results show that with increasing the nitriding time may induce N-plasma etching, which would degrade the substrate surface morphology, there is similar to the effect of H-plasma on the cleaning of sapphire substrate.

As can see from the Figure 3, the GaN as-grown films were deposition on glass substrate at $200 \mathrm{~min}$ under the condition of without nitrided and nitrided for $5 \mathrm{~min}$, $15 \mathrm{~min}$ and $30 \mathrm{~min}$ separately. Broken rings indicate that the GaN as-grown films deposition is polycrystalline with preferred axis. The broken streaks in (b) is clearer and thinner than that in (a), the means is that crystallization improved and grains of larger 
size[12], which achieved with the introduction of 5 min nitridation. The rings in (b) show the trend of breaking up into light spots, which indicates that the nitriding is helpful in enhancing the crystal orientation of GaN as-grown films. With increased the nitriding time, as shown in Figure 3 (c) and (d) the RHEED pattern of GaN were even exhibiting a little darker rather than clearer, which may because that after 5 min the nitriding of the substrate surface has almost completed, so with increasing the nitriding time, which may induce N-plasma etching and degrade the substrate surface morphology, there is similar to the effect of H-plasma on the cleaning of sapphire substrate [13].

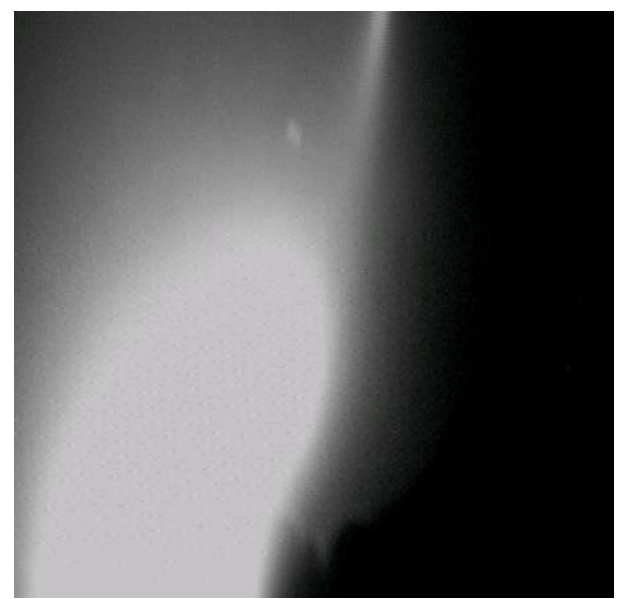

(a) without nitridin

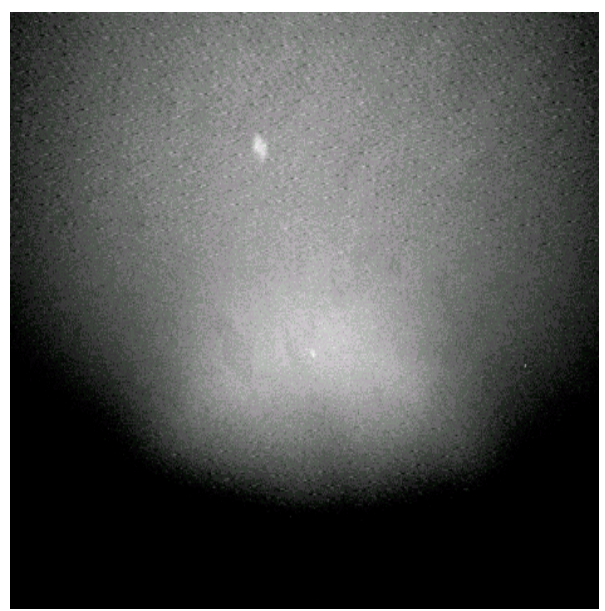

(b) nitriding

Figure 2. RHEED Patterns of Glass Substrate before and After Pure N2 Nitriding Time for 5 min

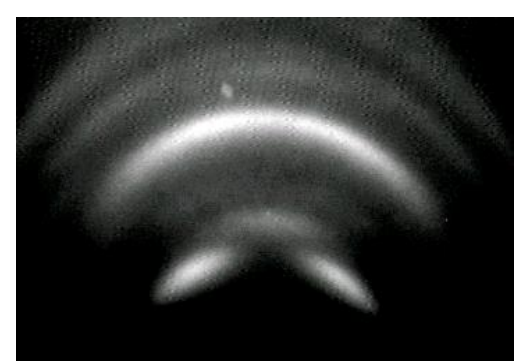

(a) 0 min

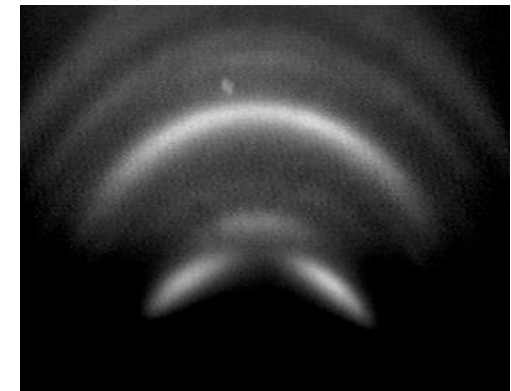

(c) $15 \mathrm{~min}$

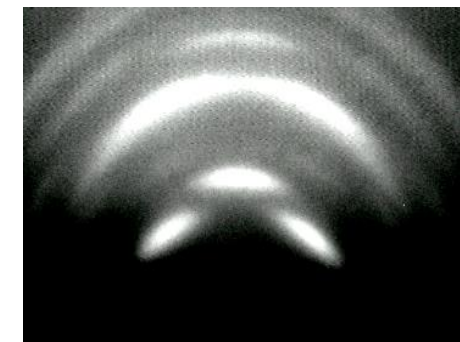

(b) $5 \mathrm{~min}$

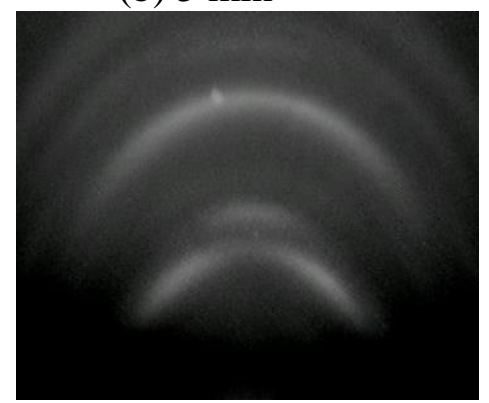

(d) $30 \mathrm{~min}$

Figure 3. GaN with Glass Substrates Nitriding Time (a) $0 \mathrm{~min}$, (b) $5 \mathrm{~min}$, (c) $15 \mathrm{~min}$ and (d) $30 \mathrm{~min}$ 


\subsection{The Structural Properties of GaN as-Grown Films}

The X-ray diffraction measurement was carried out by XRD-600 style. And the results are shown in Figure 4. The very wide and low intensity peak can be seen from $2 \theta$ about 20 to about 80 degree, which is caused by the diffraction of amorphous glass substrate. The dominant (0002) peak corresponding to $\mathrm{C}$-axis diffraction indicates that $\mathrm{GaN}$ as-grown film deposition on glass substrates is with $\mathrm{C}$-axis preferential orientation. The result is supported by RHEED pattern observed. The GaN as-grown films (103) and (0004) peaks observed in (a) disappear from (b),(c) and (d), this results indicate that with the introducing of nitriding $\mathrm{GaN}$ deposition exhibited stronger $\mathrm{C}$-axis orientation trendy. This function of nitriding is of significant importance, There as the majority of commercial optoelectronic devices are based on $\mathrm{C}$-axis oriented $\mathrm{GaN}$ thin films[3].The X-ray diffraction intensity decreased upon increasing the nitriding time from 5 to 15 and $30 \mathrm{~min}$, which are relate to the deterioration of substrate surface morphologies caused by $\mathrm{N}$-plasma etching.

Figure 4 shows the nitridation time dependence of XRD spectra for the as-grown films. The GaN (0002) peak at 34.56 is clear seen in the films deposited under the condition of various nitridation time, which can be seen weak (103) and (0004) peaks accompanying predominant (0002) peak under the high and low nitridation time, such as nitridation time of 30min. This result indicates (0002) as strong c-axis preferential orientation for films produced under this condition. However, the XRD intensity of as-grown deposited GaN films is lower than that of $\mathrm{GaN}$ films grown on $\mathrm{Si}$ because of the amorphous nature of glass substrate in the present experiments. The full width at half maximum (FWHM) with the increasement of nitridation time were found to be $0.27^{\circ}, 0.27^{\circ}, 0.29^{\circ}$, and $0.48^{\circ}$, respectively. The average grain size can be estimated by Scherrer formula as

$$
L=0.91 \lambda / D \cos \theta
$$

where $\lambda$ is the wavelength of X-ray, the FWHM of the (0002) diffraction peak, and $\theta$ the Bragg angle. Substituting these values in the above equation (1), the average grain sizes were found to be $361,40,41$, and $36 \mathrm{~nm}$, respectively, which showed in table 2 . The stress in the plane of $\mathrm{GaN}$ film with a hexagonal crystal structure can be expressed as

$$
\sigma=\frac{2 c_{13}^{2}-c_{3{ }_{3}} c+_{11} c}{2 c_{13}} \frac{)_{1}-c}{c_{0}}=-898 \frac{c-c_{0}}{c_{0}}(\mathrm{Gpa})
$$

Due to the single crystalline $\mathrm{GaN}$, the values of the elastic constant is $\mathrm{c} 11=390$ Gpa, c33=398 Gpa, c12=145 Gpa, c13=106 Gpa, respectively, c and c0 is the $\mathrm{c}$ axis crystal lattice constant of the deposited GaN film under this study and ideal crystal material. Therefore, the stress in the plane of $\mathrm{GaN}$ film can be calculated by the equation. The results were also showed in Table 2. Under various nitridation styles, the stress in the plane of GaN films all exhibit compressed stress. It is found that the stress has an increase behavior with the increasement of nitridation time, and decrease at nitridation time of $15 \mathrm{~min}$. The stress in the plane of $\mathrm{GaN}$ film has a minimum value of $-0.79 \mathrm{Gpa}$ at optimum nitridation time of $15 \mathrm{~min}$, which has the best crystallinity for $\mathrm{GaN}$ as-grown films. 


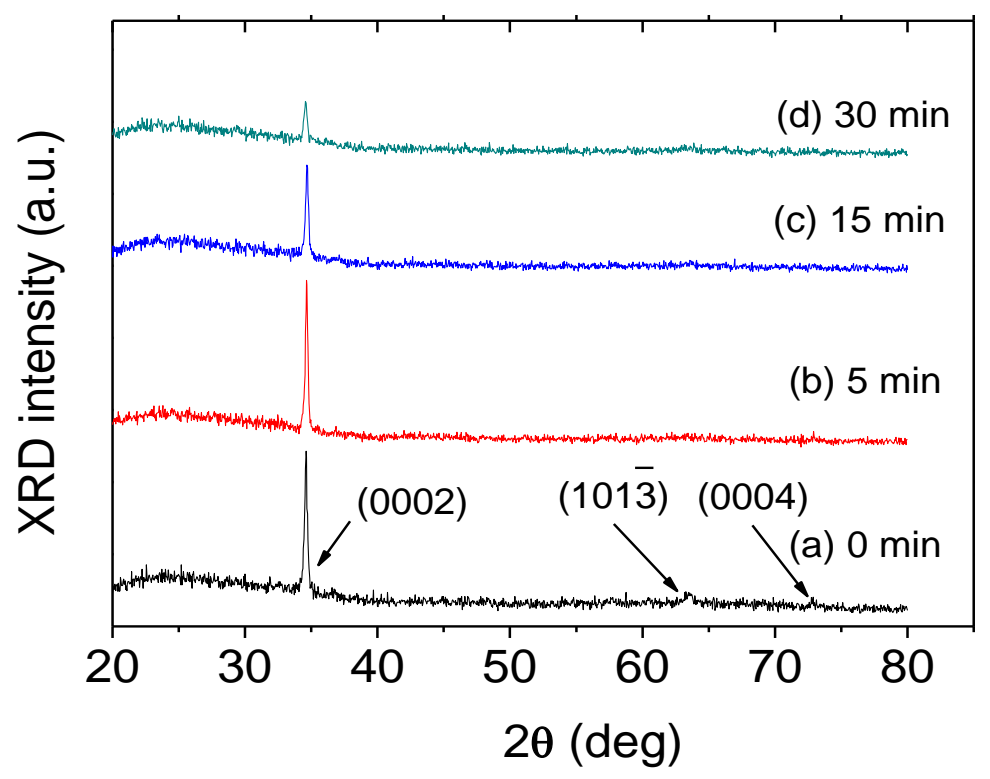

Figure 4. XRD-Spectrum of GaN Deposition with Glass Substrates without and with Nitriding Time of 5, 15 and $30 \mathrm{~min}$

Table 2. The Results of the XRD For As-Grown Films With The Nitriding Time

\begin{tabular}{cccccc}
\hline \hline Sample & $2 \theta$ & FWHM & $\begin{array}{c}\mathrm{L} \\
/ \mathrm{nm}\end{array}$ & $\begin{array}{c}\mathrm{d} \\
/ \mathrm{nm}\end{array}$ & $\begin{array}{c}\text { Stress } \\
/ \mathrm{GPa}\end{array}$ \\
\hline \hline $\mathrm{a}$ & 34.51 & 0.238 & 36 & 0.2596 & -1.89 \\
$\mathrm{~b}$ & 34.53 & 0.225 & 40 & 0.2594 & -1.12 \\
$\mathrm{c}$ & 34.55 & 0.219 & 41 & 0.2595 & -0.79 \\
$\mathrm{~d}$ & 34.51 & 0.259 & 36 & 0.2597 & -1.35 \\
\hline \hline
\end{tabular}

\subsection{Morphological Properties}

As can be seen from the Figure 5, the GaN as-grown films have exhibited rounds and island morphology, which are noticeable changes with different substrate nitriding time. The average grain size of different as-grown films is about $160 \mathrm{~nm}$ (a), after nitriding for $5 \mathrm{~min}$ as shown in (b) increased to about $240 \mathrm{~nm}$. The result is that with increased the nitriding time to $30 \mathrm{~min}$ the surface morphology deteriorated, the array of GaN grains totally out of order with amorphous size. But with the increase of nitriding time the surface morphology deterioration which may caused by the negative effect of plasma etching. Surface root mean square (RMS) roughness increase abruptly from 0.681(a) to 1.902 (b) and 1.954(c) nm, wnich confirms the N-plasma effect discussed above. 


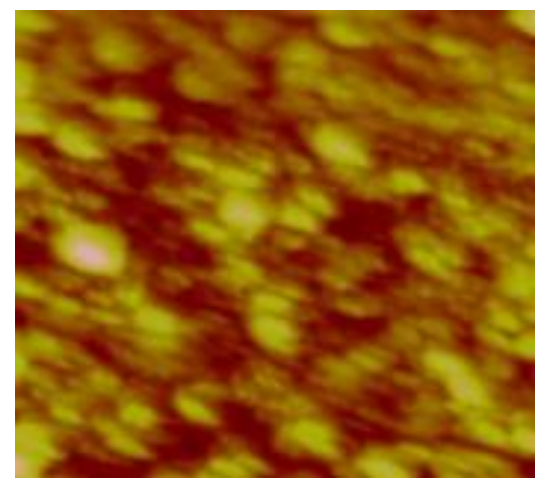

(a) 0 min

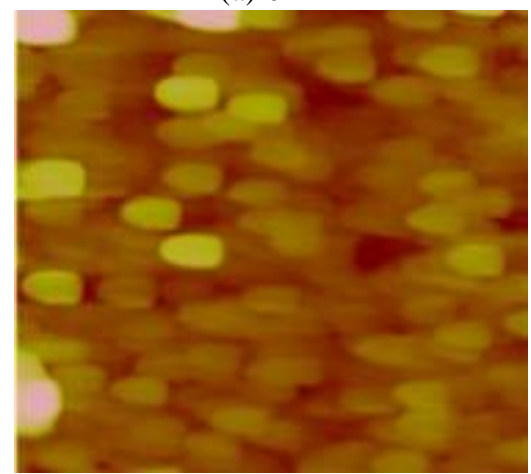

(c) $15 \mathrm{~min}$

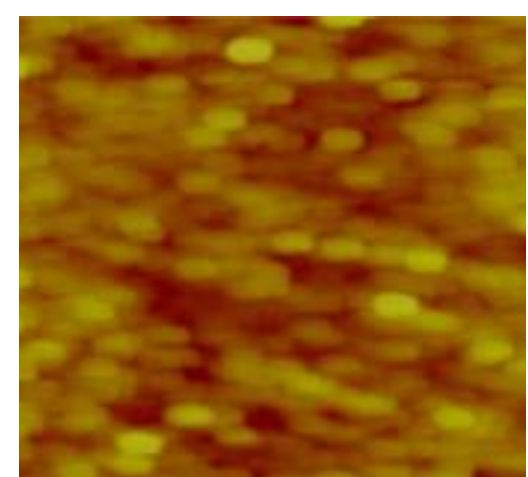

(b) $5 \mathrm{~min}$

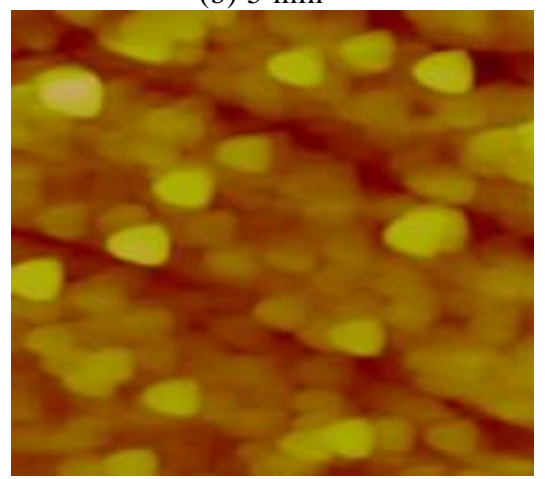

(d) $30 \mathrm{~min}$

Figure 5. AFM images $(2 \mu \mathrm{m} \times 2 \mu \mathrm{m})$ of GaN Deposition with Different Nitriding Time: (a) $0 \mathrm{~min}$, (b) $5 \mathrm{~min}$, (c) $15 \mathrm{~min}$, (d) $30 \mathrm{~min}$

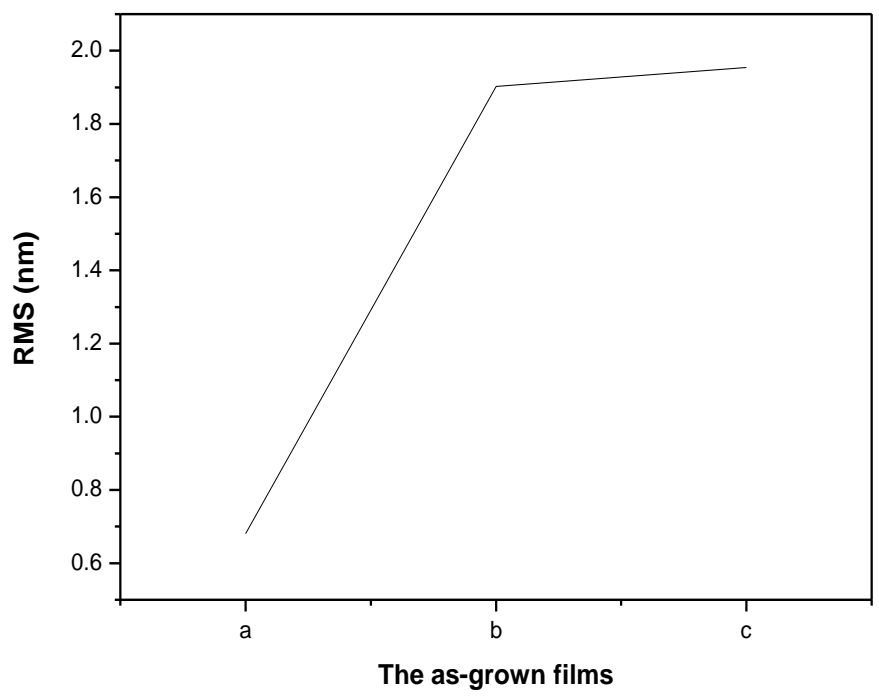




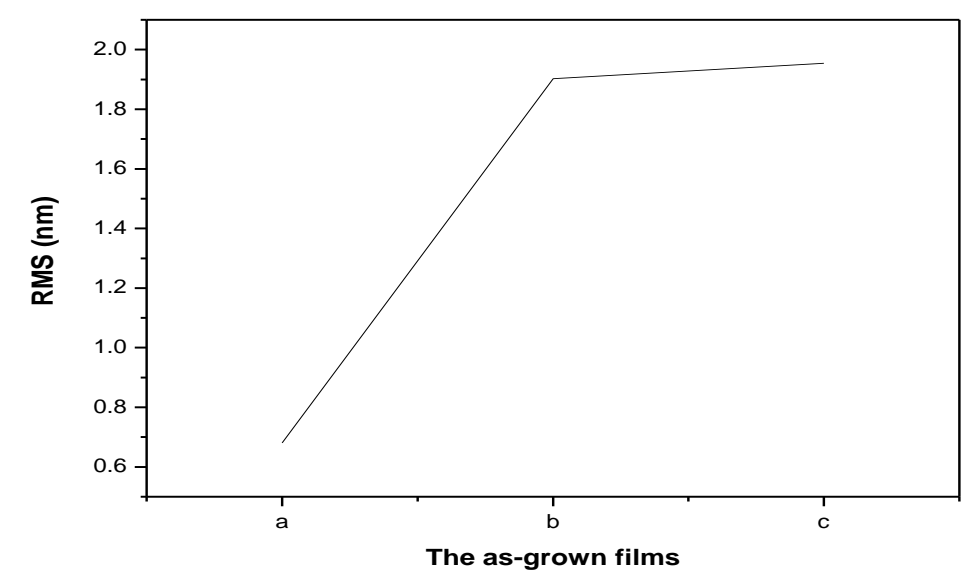

Figure 6. Surface Root Mean Square (RMS) Roughness of GaN Deposition With Different Nitriding Time: (a) 0 min,(b) $15 \mathrm{~min}$,(c) $30 \mathrm{~min}$

\subsection{Hall-Effect Properties Measurement}

The experiments were using the ECR-PEMOCVD method to deposition the high c-axis preferred orientation GaN films on amorhpous quartz glass at low temperature. The electrical properties of as-deposited films were investigated by Hall-effect measurements; the results show that all the samples were the n-type electrical behavior. It was found that mobility increased from 1.4 to $6.5 \mathrm{~cm} 2 / \mathrm{V} \cdot \mathrm{s}$ and electron concentration increased from $4.3 \times 10^{17}$ to $6.2 \times 10^{18} \mathrm{~cm}^{-3}$ as the nitriding time increased. Table 3 and Figure 7 show the hall-effect results.

Table 3. The Results of Electrical Properties for As-Grown Films with the Various Nitriding Time

\begin{tabular}{|c|c|c|c|c|}
\hline $\begin{array}{l}\text { Sample } \\
\text { Number }\end{array}$ & V / III ratio & $\begin{array}{l}\text { RMS } \\
(\mathbf{n m})\end{array}$ & $\begin{array}{l}\text { Hall mobility } \\
\left(\mathrm{cm}^{2} / \mathbf{V} \cdot \mathbf{s}\right)\end{array}$ & $\begin{array}{c}\text { Carrier concentration } \\
\left(\mathrm{cm}^{-3}\right)\end{array}$ \\
\hline$a$ & 3360 & 0.681 & 1.4 & $1.93 \times 1018$ \\
\hline b & 4200 & 1.902 & 4.2 & $2.42 \times 10^{18}$ \\
\hline c & 5600 & 1.954 & 6.2 & $5.63 \times 10^{18}$ \\
\hline d & 5600 & 1.954 & 6.2 & $0.21 \times 10^{18}$ \\
\hline
\end{tabular}




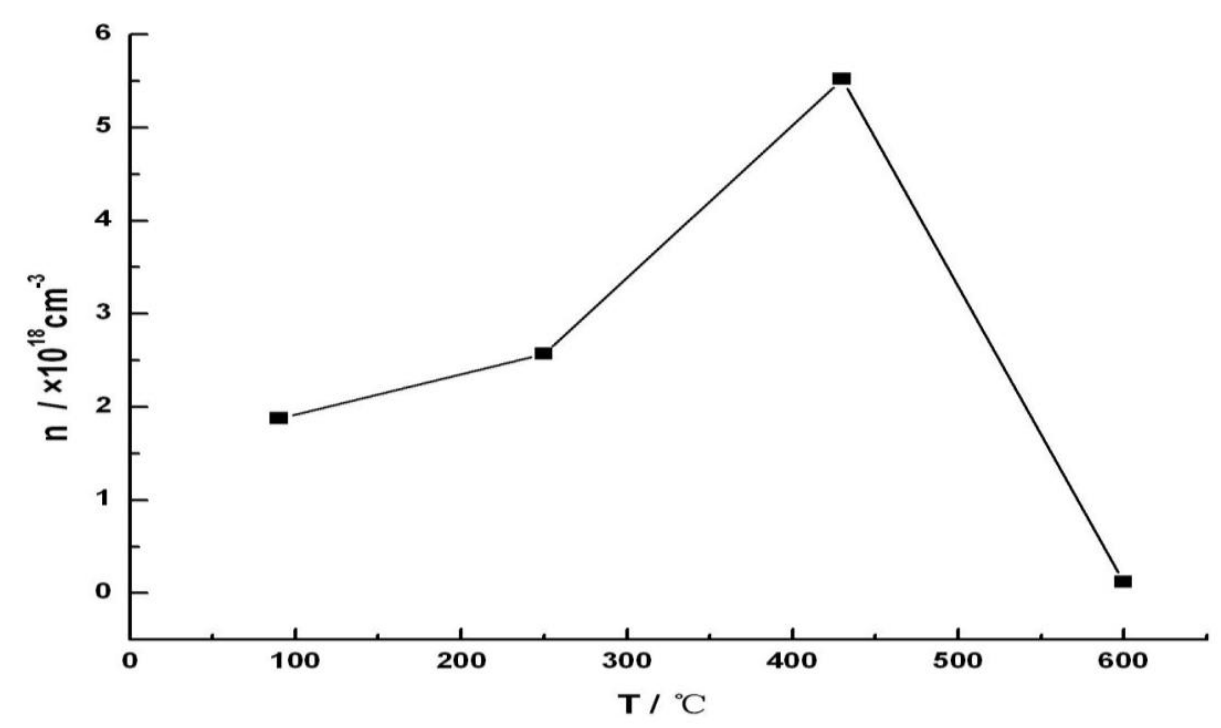

Figure 7. the Results of Electrical Properties for As-Grown Films with the Various Nitriding Time

\section{Conclusion}

1) Concentric diffraction RHEED rings demonstrated the successful nitriding time of conventional glass substrates using the self developed RHEED equipped ECR-PEMOCVD systems at low temperature $\left(430^{\circ} \mathrm{C}\right)$ far below the strain point of glass.

2) After the introduction of nitridation, the $\mathrm{GaN}$ as-grown films deposition exhibited stronger c-axis orientation, and the average grain size increased significantly with nitriding time.

3) There are increasing the nitriding time to $30 \mathrm{~min}$ the deposition deteriorated, which means that the quality of GaN deposition as-grown films depends so much on the nitriding time with 15 min was proved to be the optimum time.

\section{Acknowledgement}

This work was supported by the Natural Science Foundation of China (Grant Nos. $61372195,61371200,11401392$ ) and the Ministry of Education Key Laboratory project (LABKF1406), and the Scientific Research Fund of Liaoning Provincial Education Department (Grant No. L2015377).

\section{References}

[1] W.L. Wang, W.J. Yang, H.Y. Wang, Y.N. Zhu, M.J. Yang. "A comparative study on the properties of c-plane and a-plane GaN epitaxial films grown on sapphire substrates by pulsed laser deposition", Vacuum, (2016), pp.158-165.

[2] Q.X. Gao, R. Liu and H.D. Xiao, "Anodic etching of $\mathrm{GaN}$ based film with a strong phase-separated InGaN/GaN layer: Mechanism and properties", Applied Surface Science, (2016), pp.406-411.

[3] M.X. Zhang, Y.Q. Wang and F. Teng, "A photoelectrochemical type self-powered ultraviolet photodetector based on GaN porous films", Materials Letters, (2016), pp.117-120.

[4] Y.C. Lin, Ikai Lo and Y.C. Wang, "Characterization of M-plane GaN thin films grown on misoriented $\gamma$-LiAlO2 (100) substrates", Journal of Crystal Growth, June, (2016). 
[5] M. Reisinger, J. Zalesak and R. Daniel, "Cross-sectional stress distribution in AlxGa1-xN heterostructure on $\mathrm{Si}$ (111) substrate characterized by ion beam layer removal method and precession electron diffraction", Materials and Design, (2016), pp.476-481.

[6] A.Y. Polyakov, N.B. Smirnov and E.B. Yakimov, "Deep traps determining the non-radiative lifetime and defect band yellow luminescence in n-GaN", Journal of Alloys and Compounds (2016), doi: 10.1016/j.jallcom. June, (2016).

[7] H.Y. Wang, W.L. Wang and W.J. Yang, "Effect of residual stress on the microstructure of GaN epitaxial films grown by pulsed laser deposition", Applied Surface Science, (2016), pp.414-421.

[8] W.J. Yang, W.L. Wang and H.Y. Wang, "Epitaxial growth of nonpolar GaN films on r-plane sapphire substrates by pulsed laser deposition", Materials Science in Semiconductor Processing, (2016), pp.82-89.

[9] G.J. Deng, Y.T. Zhang and Z. Huang, "Growth of high quality N-polar n-GaN on vicinal C-face n-SiC substrates for vertical conducting devices", Vacuum, (2016), pp.119-123.

[10] J.G. Lee, H.S. Kim and K.S. Seo, "High quality PECVD SiO2 process for recessed MOS-gate of $\mathrm{AlGaN} / \mathrm{GaN}$-on-Si metal-oxide-semiconductor heterostructure field-effect transistors", Solid-State Electronics, (2016), pp.32-36.

[11] C. Cachoncinlle, E. Millon and A. Petit, "High-resolution emission spectroscopy of random lasing in GaN films pumped by UV-pulsed laser", Optics Communications, (2016), pp.49-53.

[12] Y.T. Cheng, Peng Liu and J.J. Wu, "High uniform growth of 4-inch GaN wafer via flow field optimization by HVPE", Journal of Crystal Growth, (2016), pp.24-29.

[13] T.H. Lin, S.J. Wang and Y.C. Tu, "Improving the performance of power GaN-based thin-film flip-chip LEDs through a twofold roughened surface", Materials Science in Semiconductor Processing, (2016), pp.69-75.

[14] F.W. Lee, W.C. Ke and C.H. Cheng, "Influence of different aspect ratios on the structural and electrical properties of GaN thin films grown on nanoscale-patterned sapphire substrates", Applied Surface Science, (2016), pp.223-229.

[15] S. Chromik, M. Sojková, V. Vretenár, "Influence of GaN/AlGaN/GaN (0001) and Si (100) substrates onstructural properties of extremely thin MoS2films grown by pulsedlaser deposition", Applied Surface Science, (2016).

[16] K. Wang, Y.H. Xing and J. Han, "Influence of the TMAl source flow rate of the high temperature AlN buffer on the properties of GaN grown on $\mathrm{Si}(111)$ substrate", Journal of Alloys and Compounds, (2016), pp.435-439.

[17] T. Lin, Z.R. Qiu and J.R. Yang, "Investigation of photoluminescence dynamics in InGaN/GaN multiple quantum wells", Materials Letters, (2016), pp.170-173.

[18] X.F. Han, J.H. Lee and Y.J. Lee, "Numerical analysis on the origin of thickness unevenness and formation of pits at GaN thin film grown by HVPE", (2016), pp.66-73.

[19] S. Alamé, A.N. Quezada and D. Skuridina, "Preparation and structure of ultra-thin GaN (0001)layers on In0.11Ga0.89N-single quantum wells", Materials Science in Semiconductor Processing, (2016).

[20] W.L. Wang, W.J. Yang and G.Q. Li, "Quality-enhanced GaN epitaxial films grown on (La,Sr)(Al,Ta)O3 substrates by pulsed laser deposition”, Materials Letters, (2016), pp.52-55.

[21] X.X. Li, J.M. Bian and M.H. Wang, "Realization of nitride-oxide based p-n heterojunctions with the n-VO2/ p-GaN/sapphire structure", Materials Research Bulletin, (2016), pp.199-204.

[22] W.L. Wang, W.J Yang and G.Q. Li, "Quality-enhanced GaN epitaxial films grown on (La,Sr)(Al,Ta)O3 substrates by pulsed laser deposition”, Materials Letters, (2016), pp.52-55.

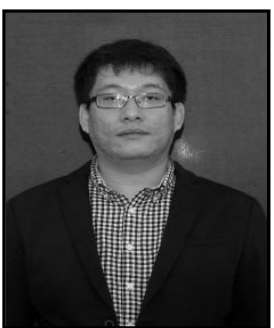

\section{Authors}

Dong Zhang, he received the Doctor's degree in microelectronics and solid-state electronics from Dalian University of technology, Dalian, China, in 2013. He works in the New Energy Institute of Shenyang Institute of Engineering as a lecturer. His research interests include the thin film materials, functional materials, the solar cell materials etc. 

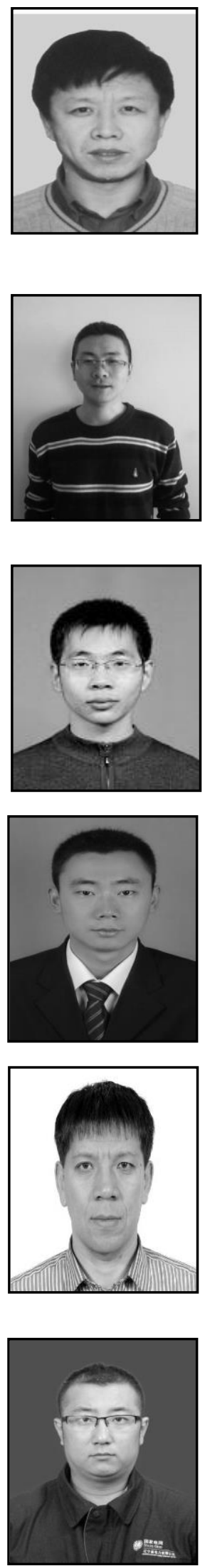

Zhenhe Ju, he received the master' degree in Solar energy engineering from Högskolan Dalarna University, Sweden, in 2004. He works in the New Energy Institute of Shenyang Institute of Engineering as a professor. His research interests include photovoltaic power generation, combined with heat and power generation etc.

Yucai Li, he received the Master's degree in Particle physics and nuclear physics from Shenyang Normal University, Shenyang, China, in 2007. He works in the New Energy Institute of Shenyang Institute of Engineering as a lecturer. His research interests include the thin film materials, micro materials, the solar cell materials etc.

Shiwei Song, he received the Doctor's degree in microelectronics and solid-state electronics from Dalian University of technology, Dalian, China, in 2013. He works in the New Energy Institute of Shenyang Institute of Engineering as a lecturer. His research interests include the thin film materials, LED, the solar cell materials etc.

Jian Wang, he received the Master's degree from Dalian University of technology, Dalian, China, in 2012. He works in the New Energy Institute of Shenyang Institute of Engineering. His research interests include the thin film materials, functional materials, the solar cell materials etc.

Gang Wang, he received his M.S. degree in Electronic Science from Shenyang Agricultural University, China, in 2011. He works in the New Energy Institute of Shenyang Institute of Engineering as a lecturer. His main research interests include pattern recognition, new material, and automatic control.

Zhenya Guo, he works in Jinzhou Power Supply Branch, State Grid Liaoning Electric Power Supply Co. Ltd. as an engineer. 
International Journal of Control and Automation

Vol. 10, No. 1 (2017) 